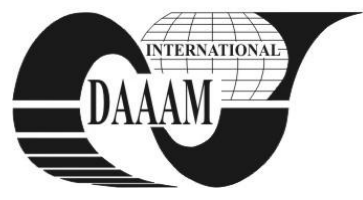

\title{
ELIMINATION OF HIGH ORDER HARMONICS FROM THE OUTPUT VOLTAGE OF AN INVERTER USING A SPECIAL CONFIGURATION DEVICE
}

\author{
IONESCU, G[elu]; PALTANEA, G[heorghe]; PALTANEA, V[eronica]; \\ NEMOIANU, I[osif] V[asile] \& CAZACU, E[mil]
}

\begin{abstract}
In the aeronautical industry it is essential to use good inverter devices to obtain a sinusoidal three phase output voltage. The output voltage of those inverters has a large content of high-order harmonics. The amplitude of those harmonics must be reduced or, ideally, totally eliminated. In this paper we propose a method to achieve this goal by using a Star-Zigzag connection winding transformer. The main benefits of using this approach are the significant reduction of the power electronics involved and the increase fiability of the device, in spite of the fact that it may slightly increase the total manufacturing expenses.
\end{abstract}

Key words: output voltage, inverter, harmonic components

\section{INTRODUCTION}

It is commonly known that the output voltage of an inverter device has a series of harmonic components which can affect the performance of the asynchronous motors (Seguier, 1985; Daut et al., 2006). In this respect, inverse couple and thermal overstress may appear. Consequently, the full-load operation active couple of the engine will be reduced. An inverter device providing an output voltage without high-order harmonics is a requirement that has to be realized despite of the higher costs involved (Phipps et al, 1994). First, it is specified that the output voltage of an inverter device has a symmetrical alternative function $f(t)=-f(t+T / 2)$, where $T$ is the period.

The amplitude of those components must be reduced, and if possible, even brought to zero.

The steps of the method proposed in this article can be summarized as follows:

- the output voltage of the inverter device it is generated as an odd function, and thus, the even harmonics are null (Ionescu et al., 2007);

- the three-phase separation transformers between the inverter device and the output charge has the primary windings realized in Delta connection mode, which cuts from the output voltage the $3 m$ order harmonics (Karov et al., 2005);

- for the remaining harmonics of the order $6 m \pm 1(5,7,11$, $13,17,19, \ldots)$ and of the order $12 m \pm 1$ and $12 m \pm 5$, with $m \in N$, the method of elimination is describe in the paper further on.

\section{ELECTRICAL DIAGRAM FOR THE OUTPUT TRANSFORMERS OF THE INVERTER DEVICE}

The output block of the inverter device it is realized by using two transformers:

- transformer 1 has the primary windings connected in Delta configuration and the secondary windings in Star configuration;

- transformer 2 has the primary windings connected in Delta configuration (with the input voltages having a phase shift of $30^{\circ}$ from the input voltages of the transformer 1) and the secondary windings in zigzag (Z) configuration (see Fig. 1) (Karov et al., 2005).

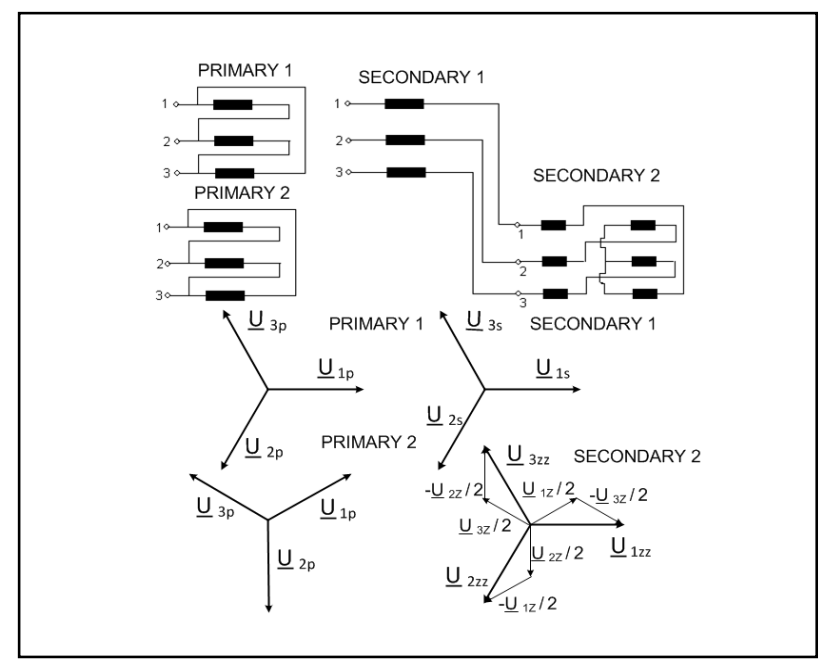

Fig. 1. The three-phase output transformers connection diagram and vector representation of the voltages

\section{THE FIRST ORDER HARMONIC COMPONENT}

Voltages induced in secondary 1 of the transformer are:

$$
\begin{aligned}
& u_{1 \mathrm{~S}}(t)=U_{\mathrm{S}} \sqrt{2} \sin (\omega t) \\
& u_{2 \mathrm{~S}}(t)=U_{\mathrm{S}} \sqrt{2} \sin (\omega t-2 \pi / 3) \\
& u_{3 \mathrm{~S}}(t)=U_{\mathrm{S}} \sqrt{2} \sin (\omega t+2 \pi / 3)
\end{aligned}
$$

Connection of secondary 2 is made in $\mathrm{Z}$ mode, and thus the output voltages are obtained from the diagram in Fig. 1, as follows:

$$
\begin{aligned}
& u_{1 \mathrm{ZZ}}(t)=\frac{u_{1 \mathrm{Z}}}{2}-\frac{u_{3 \mathrm{Z}}}{2}=\frac{U_{\mathrm{Z}}}{2} \sqrt{3} \sqrt{2} \sin (\omega t) \\
& u_{2 \mathrm{ZZ}}(t)=\frac{u_{2 \mathrm{Z}}}{2}-\frac{u_{1 \mathrm{Z}}}{2}=\frac{U_{\mathrm{Z}}}{2} \sqrt{3} \sqrt{2} \sin (\omega t-2 \pi / 3) \\
& u_{3 \mathrm{ZZ}}(t)=\frac{u_{3 \mathrm{Z}}}{2}-\frac{u_{2 \mathrm{Z}}}{2}=\frac{U_{\mathrm{Z}}}{2} \sqrt{3} \sqrt{2} \sin (\omega t+2 \pi / 3)
\end{aligned}
$$

The total output voltages are obtained by adding the voltages obtained in secondary 1 and secondary 2 :

$$
\begin{aligned}
& u_{1}=u_{1 \mathrm{~S}}+u_{1 \mathrm{ZZ}} \\
& u_{2}=u_{2 \mathrm{~S}}+u_{2 \mathrm{ZZ}} \\
& u_{3}=u_{3 \mathrm{~S}}+u_{3 \mathrm{ZZ}}
\end{aligned}
$$

One can observe that the output voltages of the two secondary transformers are in-phase, implying that the fundamental of the total voltages remains unaffected (Karov et al., 2005). 


\section{THE $6 m \pm 1$ HARMONIC COMPONENTS}

The harmonic components from the primary windings generate in the secondary ones some harmonic disturbances at the same frequency, but with the phase angle amplified by $6 m \pm 1$. For secondary 2 the output voltages are:

$$
\begin{aligned}
& u_{1 \mathrm{ZZ}}^{(6 m \pm 1)}=U_{\mathrm{Z}}^{(6 m \pm 1)} \sqrt{3} \sqrt{2} \sin [(6 m \pm 1) \omega t+m \pi] \\
& u_{2 \mathrm{ZZ}}^{(6 m \pm 1)}=U_{\mathrm{Z}}^{(6 m \pm 1)} \sqrt{3} \sqrt{2} \sin [(6 m \pm 1) \omega t+m \pi \mp 2 \pi / 3](4) \\
& u_{3 \mathrm{ZZ}}^{(6 m \pm 1)}=U_{\mathrm{Z}}^{(6 m \pm 1)} \sqrt{3} \sqrt{2} \sin [(6 m \pm 1) \omega t+m \pi \mp 2 \pi / 3]
\end{aligned}
$$

The phase voltages for the secondary 1 become:

$$
\begin{aligned}
& u_{1 \mathrm{~S}}^{(6 m \pm 1)}=U_{\mathrm{S}}^{(6 m \pm 1)} \sqrt{2} \sin [(6 m \pm 1) \omega t] \\
& u_{2 \mathrm{~S}}^{(6 m \pm 1)}=U_{\mathrm{S}}^{(6 m \pm 1)} \sqrt{2} \sin [(6 m \pm 1) \omega t \mp 2 \pi / 3] \\
& u_{3 \mathrm{~S}}^{(6 m \pm 1)}=U_{\mathrm{S}}^{(6 m \pm 1)} \sqrt{2} \sin [(6 m \pm 1) \omega t \mp 2 \pi / 3]
\end{aligned}
$$

Similarly, the two output voltages are added as it was done for the fundamental harmonic. We get:

$$
\begin{aligned}
& u_{1}^{(6 m \pm 1)}=u_{1 \mathrm{~S}}^{(6 m \pm 1)}+u_{1 \mathrm{ZZ}}^{(6 m \pm 1)} \\
& u_{2}^{(6 m \pm 1)}=u_{2 \mathrm{~S}}^{(6 m \pm 1)} / 2+u_{2 \mathrm{ZZ}}^{(6 m \pm 1)} / 2 \\
& u_{3}^{(6 m \pm 1)}=u_{3 \mathrm{~S}}^{(6 m \pm 1)} / 2+u_{3 \mathrm{ZZ}}^{(6 m \pm 1)} / 2
\end{aligned}
$$

One can notice that those voltages are zero if $m$ has an odd value and $U_{S}=\frac{U_{Z}}{2} \sqrt{3}$. The use of two transformers connected as described in this article can compensate the harmonics of the order $12 m \pm 5$, but does not compensate the $12 m \pm 1$ harmonics components.

\section{THE $12 m \pm 1$ HARMONIC COMPONENTS}

The elimination of these harmonics is performed by properly adjusting the firing angles of a thyristor. By doing so, over one cycle, the thyristors output voltage is determined by the triggering commands, as one can see in the diagram show in Fig. 2.

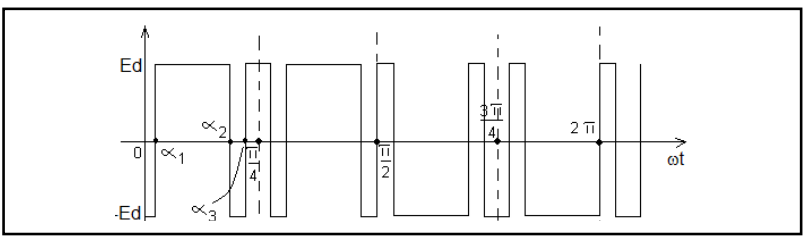

Fig. 2. The thyristors firing angle

For the first quarter of the period, the analytical expression of the function is:

$$
f(t)=\left\{\begin{array}{c}
-\frac{E_{d}}{2} \text { for } \alpha \in\left[0, \alpha_{1}\right) \\
\frac{E_{d}}{2} \text { for } \alpha \in\left[\alpha_{1}, \alpha_{2}\right) \\
-\frac{E_{d}}{2} \text { for } \alpha \in\left[\alpha_{2}, \alpha_{3}\right) \\
\frac{E_{d}}{2} \text { for } \alpha \in\left[\alpha_{3}, \frac{\pi}{4}\right)
\end{array}\right.
$$

The $f(t)$ is an odd alternative symmetrical function, and thus it contains just odd sinusoidal harmonics. From the $12 m \pm 1$ components only the harmonics 11 and 13 are important because of their high amplitude. The rest high-order harmonics are small enough and thus can be neglected. The amplitude of those harmonic components is obtained with the expression:

$$
\begin{gathered}
b_{k}=\frac{8}{T} \int_{0}^{\pi / 4} f(t) \sin (k \omega t) d t=\frac{4}{\pi} \frac{E_{d}}{2} \frac{1}{k}\left[2 \cos \left(k \alpha_{1}\right)-\right. \\
\left.2 \cos \left(k \alpha_{2}\right)+2 \cos \left(k \alpha_{3}\right)-1\right]
\end{gathered}
$$

The main problem is to minimize this expression and if it is possible to zero it. The imposed condition is:

$$
2 \cos \left(k \alpha_{1}\right)-2 \cos \left(k \alpha_{2}\right)+2 \cos \left(k \alpha_{3}\right)-1=0
$$

A more convenient approach is to find a relation between two angles only. Let us consider that $b_{1}$ the amplitude of the first order harmonic, and by denoting $a=\frac{b_{1}}{\frac{4 \cdot \frac{E_{d}}{2}}{2}}$, we get:

$$
b_{k}=\frac{b_{1}}{a} \frac{1}{k}\left[2 \cos \left(k \alpha_{1}\right)-2 \cos \left(k \alpha_{2}\right)+2 \cos \left(k \alpha_{3}\right)-1\right]
$$

Using (10) we can minimize the amplitude of the harmonics. A numerical program which computes the angles $\alpha_{2}$ and $\alpha_{3}$ in the condition given by $\alpha_{1}=0$ was generated. The obtained results are presented in the Fig. 3, representing the variation of the angles $\alpha_{2}$ and $\alpha_{3}$ versus the amplitude of the signal.

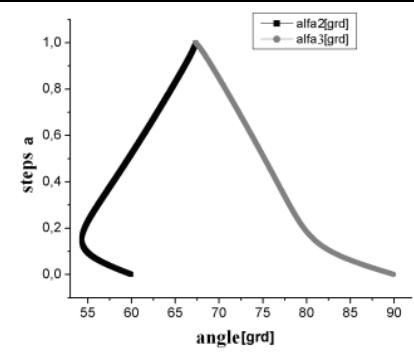

Fig. 3. The graph defining the way to minimize the $12 m \pm 1$ harmonics

\section{CONCLUSIONS}

In this paper we propose a simple and robust method that may be used in the manufacturing process of the inverter device block involved in the startup process of an airplane. The described connection of two transformers can compensate all the high-order harmonics except those of the order $12 m \pm 1$. Their amplitudes are cut off to $(0.05-0.1) \%$ of the fundamental amplitude. The even order harmonics do not appear at all due to the Delta-Star configuration. From the total odd harmonics, the $3^{\text {rd }}$ and the $9^{\text {th }}$ ones are completely eliminated due to the Star-Delta connection. The $5^{\text {th }}$ and $7^{\text {th }}$ harmonics are attenuated using the method presented in the paper, and the $11^{\text {th }}$ and $13^{\text {th }}$ harmonics are eliminated by properly adjusting the firing angle of the thyristors.

\section{REFERENCES}

Daut, I.; Syafruddin, H.S.; Rosnazri, A.; Samila, M. \& Haziah, H. (2006). The effects of harmonic components on transformer losses of sinusoidal source supplying nonlinear loads, American Journal of Applied Sciences, vol. 3, no. 12, pp. 2131-2133, ISSN: 1546-9239

Ionescu, G.; Păltânea, V.; Păltânea, G. \& Chivulescu, C. (2007). A method for elimination the $6 \mathrm{~m} \pm 1$ harmonics from the output voltage of an inverter device, Proceedings of SIITME, October, Baia Mare, Romania, ISBN 978-973713-188-1, pp. 124-126

Karov, R.; Nedeltcheva, S. \& Karov, D. (2005). Commutation and commutation factor concerning current converters connection to AC grid, Proceedings of $6^{\text {th }}$ IPSC, November, Timisoara, Romania, pp 302-306

Phipps, J.K.; Nelson, J.P. \& Sen, P.K. (1994). Power quality and harmonic distortion on distribution systems, IEEE Transaction on Industry Applications, vol. 30, no. 2, pp. 476-484, ISSN: 0093-9994

***Seguier, G. (1985). L'electronique de puissance: Les function de base et leurs principals application, Dunot, ISBN 2-04-016447-2, Paris 